\title{
Update on sepsis-associated acute kidney injury: emerging targeted therapies
}

This article was published in the following Dove Press journal:

Biologics:Targets and Therapy

7 November 2016

Number of times this article has been viewed

\section{James F Doyle' \\ Lui G Forni ${ }^{1,2}$}

'Department of Intensive Care, Medicine and Surrey Peri-Operative Anaesthesia and Critical Care Collaborative Research Group, Royal Surrey County Hospital NHS Foundation Trust, ${ }^{2}$ Faculty of Health and Medical Sciences, University of Surrey, Guildford, Surrey, UK
Correspondence: James Doyle Department of Intensive Care Medicine, Royal Surrey County Hospital NHS Foundation Trust, Egerton Road, Guildford GU2 7XX, Surrey, UK Email james.doyle4@nhs.net
Abstract: Sepsis-associated acute kidney injury (SA-AKI) is an independent predictor of increased mortality and morbidity. It is essential that further advances in the treatment of sepsis should prioritize targeted therapies in SA-AKI in order to improve these bleak outcomes. As yet, a unique therapy that effectively reduces the impact of acute kidney injury has not been demonstrated. However, the emergence of novel targeted therapies, perhaps in combination, has the possibility of significantly reducing the long-term sequelae of an episode of SA-AKI. In this review, we will focus on the shared etiology of these conditions and how this is managed with targeted therapy and finally the emerging novel therapies that may play an additional role to current treatment strategies.

Keywords: sepsis, acute kidney injury, novel targeted therapy outcome

\section{Introduction}

Acute kidney injury (AKI) and the sepsis syndrome are complex heterogeneous conditions with common risk factors. Severe sepsis and septic shock remain major causes of mortality and morbidity in intensive care units (ICUs) in developed countries, with sepsis long recognized as a precipitant of AKI. Adult patients with AKI in the context of sepsis have a mortality rate of $60 \%,{ }^{1}$ while in children, this varies from $57 \%$ to $66 \%{ }^{2}$ Furthermore, the long-term outcome of an episode of AKI during a critical illness has recently been shown to be associated with significant morbidity and mortality. Given these observations, it is essential that further advances in the treatment of sepsis should prioritize targeted therapies in sepsis-associated AKI (SA-AKI), in order to improve the bleak outcomes. Part of the reason that patients with SA-AKI appear disadvantaged is that they display a greater acuity of illness, ${ }^{3}$ manifest physiologically as lower blood pressure, tachycardia, worse pulmonary function (the ratio of partial pressure arterial oxygen to the fraction of inspired oxygen $\left[\mathrm{PaO}_{2} / \mathrm{FiO}_{2}\right]$ ) and $\mathrm{pH}$, and a higher white blood cell count. This is reflected in the higher mortality observed in SA-AKI compared to nonseptic AKI, both in the ICU and in general wards, across all stages of AKI. ${ }^{4}$

The management of sepsis has been revolutionized, particularly outside the ICU, with emphasis placed on early intervention ensuring a standard of care focusing on the effective treatment of the underlying infection. However, to date, there is no single effective therapy that has been shown to alter the outcome of SA-AKI. Rather, the process of care seems to confer a survival benefit. In this review, we will focus on the emerging targeted therapies that may play an additional role to the current treatment strategies and provide improved outcomes over the coming years. 


\section{Pathophysiology}

The pathophysiology of SA-AKI is complex above and beyond the intravascular volume status and perfusion pressure. While ischemia-reperfusion models or nephrotoxininduced renal injury may go some way toward explaining the pathogenesis for some AKI etiologies, in SA-AKI associated with a hyperdynamic circulation, the pathophysiology differs.

Recent research indicates inflammatory mediators causing tubular cell dysfunction and cell cycle arrest along with the associated microcirculatory dysfunction as the trigger for SA-AKI. ${ }^{5}$ This process is in keeping with cellular injury processes resulting in multiorgan dysfunction such as that seen in severe sepsis.

The overall pathophysiology is summarized in Figure 1.

Multiple cell lines contribute to the pathophysiology of the inflammatory response. At the microvascular level, this leads to localized stasis within blood vessels and the release of inflammatory cytokines, including the components that cause activation of the coagulation system. This is seen as an increase in the expression of microparticles (small 100-1,000 nm vesicular bodies originating from activated or apoptotic cells). In particular, an association has been found between platelet-derived microparticles and the development of SA-AKI. ${ }^{6}$

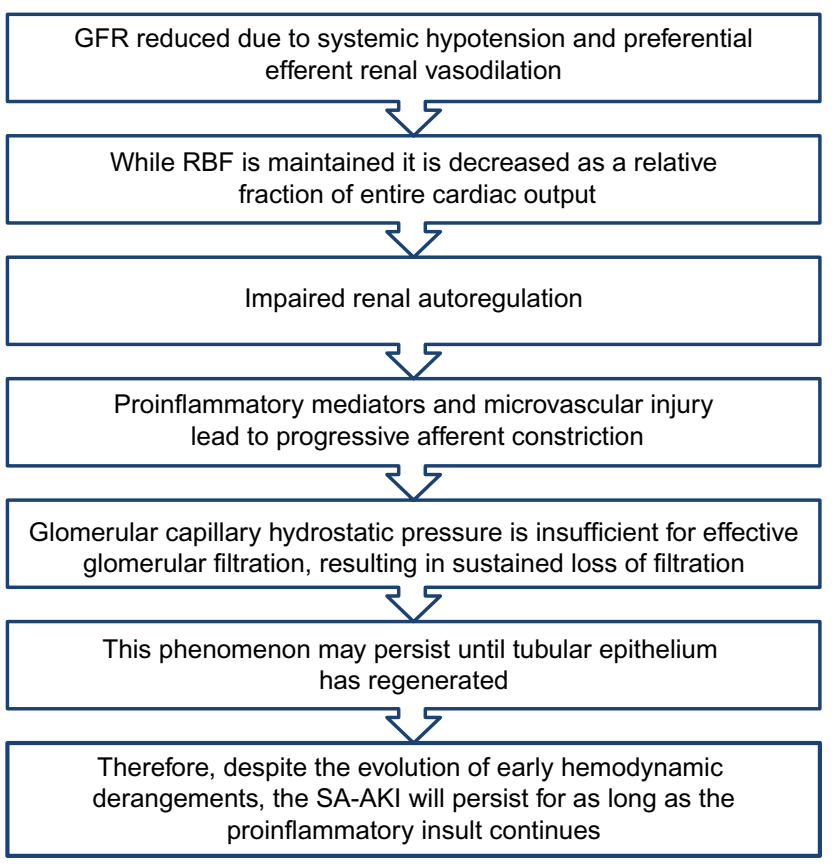

Figure I Pathophysiology of sepsis associated acute kidney injury.

Note: Data from Prowle and Bellomo. ${ }^{5}$

Abbreviations: GFR, glomerular filtration rate; RBF, renal blood flow; SA-AKI, sepsis-associated acute kidney injury.

\section{AKI assessment}

Although AKI is defined in terms of changes in serum creatinine from baseline, as well as the less-frequently measured urine output, these measures, particularly serum creatinine, are inadequate in the acute situation. Much recent research has led to the quest for novel biomarkers as an acute assessment of renal function at the onset of AKI. Indeed, biomarkers are now available in some areas of clinical practice, which are able to better predict the severity of AKI, particularly when associated with sepsis. ${ }^{7}$ The most promising of these are plasma and urinary neutrophil gelatinase-associated lipocalin and urinary interleukin (IL)18 and kidney injury molecule- $1 .^{8,9}$ These biomarkers are proteins that are upregulated in response to renal injury. Cystatin-C, a functional marker, was also a promising plasma marker, although one study of 327 patients demonstrated no signal for SA-AKI. ${ }^{10}$

\section{Sepsis assessment}

As with AKI, there is a uniform approach to the definition of sepsis. The American College of Chest Physicians and the Society of Critical Care Medicine joint statement defines sepsis and severe sepsis. ${ }^{11}$ This requires the presence of infection together with systemic manifestations, whilst severe sepsis is sepsis with sepsis-induced organ dysfunction and/ or tissue hypoperfusion.

\section{Shared susceptibility}

Shared risk factors for sepsis and AKI include the presence of immunocompromised and proinflammatory states (Table 1).

Of note is the finding that the distribution of pathogens causing SA-AKI is consistent with that of those causing severe sepsis in a general ICU setting. For nosocomial infections, Gram-positive bacterial pathogens predominate at around 50\% compared to Gram-negative bacteria at 39\% and fungal at $11 \% .^{12}$

\section{Outcome of SA-AKI}

Independent risk factors for mortality among patients with SAAKI include age, acute physiology and chronic health evaluation (APACHE II) score, duration of mechanical ventilation, duration of mean arterial pressure $<65 \mathrm{mmHg}$, commencement of renal replacement therapy (RRT), and Kidney Disease: Improving Global Outcomes (KIDGO) stage of AKI. ${ }^{13,14}$ Risk prediction for AKI is well established with independent risk factors and risk prediction scoring systems described in the literature, which can be further targeted to SA-AKI. An 
Table I Shared susceptibility

\begin{tabular}{|c|c|c|}
\hline \multicolumn{3}{|l|}{ Shared susceptibility } \\
\hline Immunocompromised & AKI & Sepsis \\
\hline Diabetes mellitus & $\begin{array}{l}\text { An independent risk factor for AKI. Most likely due to increased } \\
\text { predisposition to hypoxia }\end{array}$ & $\begin{array}{l}\text { Altered immunity with resultant predisposition to } \\
\text { infection }\end{array}$ \\
\hline Cirrhosis & $\begin{array}{l}\text { Hyperbilirubinemia, hypoalbuminemia, low intravascular volume } \\
\text { due to capillary leak, and high infection risk all predispose to AKI }\end{array}$ & $\begin{array}{l}\text { Impaired neutrophil function among other } \\
\text { alterations in immunity predisposes toward infection }\end{array}$ \\
\hline HIV & $\begin{array}{l}\text { Glomerular disease and nephrotoxicity from antiretrovirals } \\
\text { predisposes to AKI/CKD }\end{array}$ & \\
\hline Malignancy & $\begin{array}{l}\text { Incidence and severity of AKI depend on type and stage of } \\
\text { malignancy }\end{array}$ & \\
\hline \multicolumn{3}{|l|}{ Proinflammatory } \\
\hline ARDS & ARDS is associated independently with the development of AKI & \\
\hline CKD & $\begin{array}{l}\text { CKD predisposes to AKI owing to reduced renal reserves and } \\
\text { reduced clearance of cytokines. Survival for AKI secondary to } \\
\text { CKD can be better than de novo AKI (likely due to lesser insult } \\
\text { required to cause the AKI); however, long-term outcomes such } \\
\text { as dialysis dependence is worse }\end{array}$ & $\begin{array}{l}\text { Reduced renal clearance of inflammatory mediators, } \\
\text { uremia leading to phagocyte dysfunction, and } \\
\text { disruption of gastrointestinal barrier all predispose } \\
\text { toward sepsis }\end{array}$ \\
\hline Post-op & $\begin{array}{l}\text { Differing profiles for the risk of AKI have emerged following } \\
\text { assessment of different types of major surgery. Specifically, } \\
\text { there are several validated scoring systems following cardiac } \\
\text { surgery for the risk of AKI. Mortality rates for post-op AKI have } \\
\text { improved, but remain high }\end{array}$ & \\
\hline
\end{tabular}

Note: Data from Heung and Koyner ${ }^{48}$ and Leelahavanichkul et al. ${ }^{49}$

Abbreviations: AKI, acute kidney injury; ARDS, acute respiratory distress syndrome; CKD, chronic kidney disease; HIV, human immunodeficiency virus; Post-op, post-operative.

example is the adaptation of current grading systems such as the use of KDIGO classification with consecutive hours of oliguria following diagnosis of sepsis. This was performed in a retrospective analysis of 390 patients, $25 \%$ of whom developed SA-AKI ( $\geq$ stage 2 ) with consecutive oliguria for 3 hours showing a fair predictive ability for $\geq$ stage 2 AKI (area under receiver operating characteristic curve, $0.73 ; 95 \%$ confidence interval, 0.68-0.78) and oliguria for 5 hours demonstrating optimal accuracy $(82 \%$; $95 \%$ confidence interval, $79 \%-86 \%) .{ }^{15}$

With the advancement of imaging techniques including functional studies, prognostic utility for AKI is available now. One promising modality is the use of Tc-99m 2,3-dimercaptosuccinic acid renal cortical imaging, with statistically significant findings obtained in patients with SA-AKI for short-term survival (defined as discharged alive from ICU). ${ }^{16}$ However, the cost and practical implications of using this as a prognostic tool are yet to be considered.

In 2014, an observational study from the US demonstrated a worse outcome with high plasma IL-6 levels taken in patients during the first 8 hours of RRT for SA-AKI. ${ }^{17}$ Another European-based study similarly found a poor outcome with raised serum kynurenic acid, an end product of tryptophan metabolism, during early (within 96 hours) RRT for SA-AKI. ${ }^{18}$ A prospective observational study from ten centers demonstrated increased plasma neutrophil gelatinase-associated lipocalin concentrations on presentation in patients with presumed sepsis who subsequently developed SA-AKI. ${ }^{19}$ Current conventional markers of inflammation, such as lactate, C-reactive protein, or procalcitonin, have not been shown to have this predictive power, with these markers decreasing slowly over time in both survivors and nonsurvivors. The use of an early predictor could be used as part of a protocol to tailor targeted therapies, although there are concerns that these may reflect the sepsis process in general, rather than specifically SA-AKI.

Importantly, there is increasing evidence that reversal of SA-AKI early (within 24 hours) corresponds to a survival benefit, when compared to persistent AKI, new AKI, and even in the absence of AKI. This was shown in a 2014 observational study by analysis of the Cooperative Antimicrobial Therapy of Septic Shock database from a cohort of over 5,000 patients in Canada, US, and Saudi Arabia. ${ }^{20}$ This has two important ramifications: firstly, the targeted therapy to reverse SA-AKI may confer improved outcome and secondly, the ability to identify these patients and use of this data may add to the accuracy of predictive scoring systems.

\section{Targeted therapies}

It is worth considering the importance of current practice with regard to SA-AKI. After several reiterations of the surviving 
sepsis guidelines, there has been a significant reduction in mortality for all-comers with sepsis; however, this treatment has not translated into a beneficial reduction of the incidence or severity of AKI. Also, there are several studies which demonstrate that the use of early goal-directed therapy does not affect the incidence or outcome of AKI. ${ }^{13}$ With regard to potential therapies for AKI, these may be classified as those targeted to restore homeostatic balance (usual therapies) and novel therapies aimed specifically at SA-AKI.

\section{Usual therapies \\ Fluids \\ Composition}

The type of fluid used in the management of sepsis has been topical for some time with the results of quality research demonstrating a shift in global clinical management. Key events can be summarized as follows:

- 2008; VISEP - A crossover study of pentastarch and intensive insulin therapy. In this study, hydroxyethyl starch (HES) was associated with increasing AKI and need for RRT. ${ }^{21}$

- 2011; SAFE substudy - Possible improved mortality outcome with albumin. ${ }^{22}$

- 2012; 6S study - HES 130/0.4 versus Ringer's lactate in severe sepsis. Patients receiving HES had an increased risk of death and need for RRT. ${ }^{23}$

- 2012; CHEST - Trend toward increased mortality with $6 \%$ HES (Voluven 130/0.4) compared to $0.9 \%$ saline and significantly increased requirement of RRT with HES. ${ }^{24}$

- 2015; Split Trial - No difference between saline and balanced solutions in development of $\mathrm{AKI}{ }^{25}$

Despite this, other than optimizing fluid therapy for sepsis and good husbandry of critical care electrolyte management, there is a paucity of specific evidence for any fluid choice in SA-AKI except avoiding synthetic starches.

\section{Positive fluid balance}

A late positive fluid balance is an independent risk factor for mortality in severe sepsis. However, this is not associated with either protection against or risk of AKI. ${ }^{26}$ This was most recently confirmed in a retrospective analysis of SA-AKI with determination of fluid balance at onset of organ dysfunction, vasopressor initiation, and sepsis diagnosis.

\section{Vasopressors}

In the setting of a replete intravascular volume, the maintenance of an adequate mean arterial pressure of $65 \mathrm{mmHg}$ in a patient without preexisting hypertension aids in adequate endorgan perfusion. Also, the current sepsis guidelines suggest the use of noradrenaline as first-line therapy with vasopressin as a second-line agent. Of interest is evidence from animal models demonstrating that vasopressin causes less tubular apoptosis, systemic inflammation, and renal damage, when compared to noradrenalin. ${ }^{27}$ The VANISH trial (VAsopressin vs Noradrenaline as Initial therapy in Septic sHock: ISRCTN20769191) has now completed recruitment while the results are awaiting publication. ${ }^{28}$ Recent scientific meetings detail that the vasopressin group had reduced total pressor requirements and reduced need for RRT, however, with no change in the primary outcome, the number of renal failure free days as defined by stage $3 \mathrm{AKI}$.

\section{Antimicrobial selection}

It is emerging that in the event of SA-AKI, the risk-benefit profiling of antimicrobial choice may need to consider renal outcome also. This is evidenced recently by a German-based retrospective analysis of 1,159 patients which demonstrated that the use of vancomycin in patients with SA-AKI increases the probability of a prolonged need for RRT at ICU discharge.$^{29}$ Furthermore, the use of bactericidal antibiotics has been shown to be associated with a transient worsening of renal function and more inflammation in the acute period. ${ }^{30}$

\section{Renal replacement therapy \\ Timing}

There is no consensus on the optimal time to initiate RRT. Some retrospective and observational studies suggest that early implementation of RRT could improve the prognosis for these patients. However, this is not backed up by large studies. Recently, a retrospective review from the People's Republic of China demonstrated no difference in outcome with early versus late RRT. ${ }^{31}$

\section{Dosing}

The IVOIRE study showed no improvement with high-volume hemofiltration (HVHF) at $70 \mathrm{~mL} / \mathrm{kg} / \mathrm{h}$ compared to standardvolume hemofiltration (SVHF) at $35 \mathrm{~mL} / \mathrm{kg} / \mathrm{h}$ with regard to mortality, hemodynamic profile, or organ function. ${ }^{32}$ Single randomized controlled trials (RCTs) have demonstrated the same findings. ${ }^{33}$ This result was further confirmed in a metaanalysis of four trials concerning 470 participants. Pooled analysis did not show any mortality difference. Furthermore, adverse events such as hypophosphatemia and hypokalemia were more common with $\mathrm{HVHF}(>50 \mathrm{~mL} / \mathrm{kg} / \mathrm{h}) .{ }^{34}$ A recent Cochrane review pooled three much smaller trials and again found no benefit of HVHF. ${ }^{35}$ However, a basic tenet in the 
survival from sepsis is early source control and appropriate antibiotic therapy. In many "high-volume" studies, no correction was made for antibiotic flux, and hence, patients may have been effectively underdosed.

Mode

Although there is evidence that continuous venovenous hemodiafiltration (CVVHDF) results in improved proinflammatory cytokine removal with a recent study showing reduced levels of vascular endothelial growth factor and other cytokines along with improved renal recovery when using CVVHDF compared to continuous venovenous hemofiltration $(\mathrm{CVVH}),{ }^{36}$ there is little evidence to support one technique over another.

\section{Specialized extracorporeal techniques}

Following the disappointing progress with the timing and dosing of RRT, it has been suggested that targeted therapies may include specialized extracorporeal techniques such as high cutoff hemofilters and hemoadsorption ${ }^{34}$ Hemoadsorption for enhanced endotoxin and cytokine removal has been studied. Results from a prospective case series in 2013 demonstrated a significant reduction of $37 \%$ in Sequential Organ Failure Assessment scores within 48 hours of initiation of CVVH with hemoadsorption, compared to a rise of $3 \%$ in Sequential Organ Failure Assessment scores in those without hemoadsorption. ${ }^{37}$ Extracorporeal therapy with polymyxin B reduced the proapoptotic activity in patients with sepsis. ${ }^{27}$ To date, a mortality benefit has not been shown, as evidenced by a larger RCT of polymyxin B from France. ${ }^{38}$

Perhaps moving on from targeted cytokine removal would be the ability to replace the endocrine and metabolic functions of the kidney beyond that of RRT. This may be achievable with bioartificial renal epithelial cell system therapy, which has been developed as a standard hemofiltration cartridge with human renal tubular cells attached along the inner surface of hollow fibers. Such renal tubule assist devices (RAD) have been the subject of a Phase II trial. This multicenter trial involving 59 patients demonstrated significant mortality benefit (33\% in RAD group and 61\% in conventional RRT at 28 days), with Kaplan-Meier analysis determining a survival advantage for 180 days. In addition, RAD was associated with greater renal recovery. ${ }^{39}$ If proven to offer survival benefit in SA-AKI, the production and distribution of RAD cartridges are an obstacle yet to be overcome. ${ }^{40}$

\section{Novel therapies}

Weak evidence exists for some of the more novel targeted therapies for both sepsis and SA-AKI, which have been described later. It is unlikely that there will be a single agent that is able to drastically alter SA-AKI outcomes, although combination therapy may well confer benefit.

\section{Alkaline phosphatase}

Alkaline phosphatase has been investigated as a treatment for AKI. While the mechanism of action remains incompletely understood, the underlying principle is dephosphorylation of endotoxins and attenuation of the inflammatory response. A prospective, randomized, double-blind, placebo-controlled trial in 2012 demonstrated a clear improvement in endogenous creatinine clearance $(P=0.02)$ along with reduced requirement and duration of RRT after 28 days. ${ }^{41}$ The same study also measured urinary biomarkers and demonstrated significant decreases in kidney injury molecule-1 and IL-18, both described to have prognostic utility for RRT requirement. This followed from earlier studies which demonstrated the administration of alkaline phosphatase was associated with reduced expression of inducible nitric oxide synthase in the proximal tubule. Most recently, another Phase I trial to assess the pharmacokinetic profile and safety of recombinant alkaline phosphatase demonstrated an acceptable profile, thus enabling progress to further interventional studies in $\mathrm{SA}-\mathrm{AKI}{ }^{42}$ This is currently recruiting.

\section{Erythropoietin}

Erythropoietin decreases apoptotic cell death. So, given the known pathophysiology of SA-AKI, it is a reasonable therapy to investigate. However, high-dose erythropoietin is known to cause vasoconstriction and an increased risk of thrombosis. Newer erythropoietins have no hematopoietic activity. This perhaps may translate into benefit for the SA-AKI patients.

\section{Methylene blue}

Methylene blue has the effect of scavenging nitric oxide (NO) and inhibiting nitric oxide synthase. During proinflammatory states, the induction of NO is associated with proximal tubular injury. Small ICU studies in the Netherlands demonstrate that short-term methylene blue infusion is associated with reduced NO production and reduced urinary excretion of renal tubular injury markers. ${ }^{43}$

\section{Molecular approach}

Human studies targeting single proinflammatory cytokines, for example, antitumor necrosis factor- $\alpha$, IL- $1 \beta$, and Toll-like receptor 4 , inhibition have not met with success. ${ }^{44}$ However, dual targeted strategy with IL- $1 \beta$ and IL-18 could be a potential treatment option for sepsis with the recent animal studies 
Table 2 Other emerging novel therapies

\begin{tabular}{|c|c|}
\hline Potential therapy & Description/trial progress \\
\hline PPAR- $\alpha$ & Inhibition of proinflammatory molecules, including IFN and IL- I $7^{44}$ \\
\hline MSCs & $\begin{array}{l}\text { Used for some time in hematology-oncology, MSCs promote bone marrow recovery and transplant tolerance. In sepsis, } \\
\text { MSCs reduce inflammation and } \mathrm{AKI}^{47}\end{array}$ \\
\hline $\begin{array}{l}\text { Anti-inhibitory PD-I } \\
\text { and its ligand (PD-LI) }\end{array}$ & $\begin{array}{l}\text { PD-I and PD-LI could contribute to immunosuppression in late sepsis. In animal models of sepsis, the use of anti-PD-I and } \\
\text { anti-PD-LI improved survival }{ }^{50}\end{array}$ \\
\hline Desferrioxamine & $\begin{array}{l}\text { Iron chelation therapy. Iron can cause cellular injury by inducing oxidative radicals. Pathogenic microbials secrete } \\
\text { siderophores which chelate iron so impairing cellular processes. Therefore, microbial "iron starvation" is a potential } \\
\text { strategy to counter sepsis }\end{array}$ \\
\hline Hepcidin & This agent also sequesters iron, with several studies demonstrating renal protection ${ }^{40}$ \\
\hline 2-Methoxyestradiol & $\begin{array}{l}\text { This anticancer drug inhibits HIF-I } \alpha \text { and NF- } \beta \text {. HIF, released as a consequence of sepsis, induces hypoxia and promotes } \\
\text { proinflammatory cytokines. In animal models, } 2 \text {-methoxyestradiol reduced mortality and SA-AKI complications } s^{51}\end{array}$ \\
\hline Resveratrol & $\begin{array}{l}\text { A scavenger of reactive nitrogen species, with studies demonstrating diminished tubular oxidative stress, improved } \\
\text { microcirculatory flow, prevention of SA-AKI, and improved survival }{ }^{51}\end{array}$ \\
\hline Ethyl pyruvate & $\begin{array}{l}\text { Another antioxidant and free radical scavenger. Ethyl pyruvate reduced both mortality and AKI in animal models. Currently } \\
\text { in Phase II trials }{ }^{52}\end{array}$ \\
\hline Anthracycline & $\begin{array}{l}\text { A known chemotherapy drug. Reduced complications of sepsis owing to autophagy, the process of clearance of cellular } \\
\text { debris }^{44}\end{array}$ \\
\hline$\alpha-M S H$ & A melanocortin agonist. This has been shown to have anti-inflammatory and antiapoptotic activities ${ }^{40,53}$ \\
\hline $\begin{array}{l}\text { SIP analogs, adenosine } \\
\text { analogs, inducible NOS } \\
\text { inhibitors, fibrates }\end{array}$ & $\begin{array}{l}\text { These drugs have the potential to reduce injury for ischemic reperfusion injury. Whether or not this would translate into } \\
\text { any protection against SA-AKI remains to be } \operatorname{seen}^{52}\end{array}$ \\
\hline $\begin{array}{l}\text { Products of heme } \\
\text { catabolism, HO-I }\end{array}$ & Stress responsive HO-I enzyme system has important antioxidant, anti-inflammatory, and antiapoptotic properties ${ }^{54}$ \\
\hline
\end{tabular}

Abbreviations: $\alpha-M S H, \alpha$-melanocyte-stimulating hormone; AKI, acute kidney injury; HIF, hypoxia-inducible factor; HO-I, heme oxygenase-I; IFN, interferon; IL-17, interleukin-17; MSCs, mesenchymal stem cells; NOS, nitric oxide synthase; PD-I, programmed cell death protein I; NF- $\beta$, nuclear factor beta; PPAR- $\alpha$, peroxisome proliferator activated receptor- $\alpha$; SIP, sphingosine I phosphate; SA-AKI, sepsis-associated acute kidney injury.

demonstrating an association between this gene, survival, and organ failure-free days. ${ }^{45}$

Soluble thrombomodulin has been considered following the unsuccessful intervention of activated protein C (APC). APC was withdrawn from clinical practice as a treatment for sepsis due to significantly increased bleeding events. APC was thought to prevent thrombosis and, thus, maintain adequate regional microcirculation. Thrombomodulin is a cofactor in generating APC and its use is thought to prevent regional thrombosis without causing systemic effects. The mechanism behind the prevention of AKI is thought to be related to reduced leukostasis and an anti-inflammatory effect owing to endothelial cell signaling. This AKI prevention may be independent of the anticoagulant effect. Clinical studies to show the efficacy in sepsis and SA-AKI are in progress (Phase 3 Safety and Efficacy Study of ART-123 in Subjects With Severe Sepsis and Coagulopathy; NCT01598831).

\section{Immunomodulation}

1,25-Dihydroxycholecalciferol upregulates cathelicidin. This has been shown to be a broad-spectrum antimicrobial polypeptide. Those animals with overexpression of this polypeptide have reduced susceptibility to bacterial infection. Unfortunately, several RCTs (a Phase IIa and a Phase II trial) have not reported any survival benefit with dosing of 1,25-dihydroxycholecalciferol in sepsis. ${ }^{46}$

Ghrelin, a neuropeptide, shows a protective effect on kidneys by inhibiting proinflammatory cytokines, particularly tumor necrosis factor- $\alpha$, in the kidneys. Ghrelin may hold promise for managing endotoxemia-induced AKI.

CVVH has an immunomodulatory effect, with the transcriptional activity of IL-6 increasing during therapy with CVVH for sepsis. ${ }^{47}$ The issue that remains is that CVVH removes pro- and anti-inflammatory mediators in a nonselective way.

Other novel therapies have been postulated, but as yet lack evidence in the field of SA-AKI (Table 2).

\section{Conclusion}

There appears to be a growing body of evidence that sepsisassociated AKI is a pathophysiologically different entity from other types of AKI. As such, the assessment and prognostic tools used to assess severity of AKI will need to be considered carefully and there is evidence that this process has begun.

When it comes to the management of SA-AKI first principles remain those that have evidence base. Such as that proposed by surviving sepsis guidelines and the general ICU management of end-organ optimization. However, further to 
this is the emergence of targeted therapies that may improve AKI. There is no argument that SA-AKI is an independent predictor of increased mortality, and the emergence of novel therapies proves to be an exciting development. However, the importance of adequate follow-up and specialist nephrology input will complete the intervention to reduce the long-term sequelae of an episode of SA-AKI.

\section{Disclosure}

The authors report no conflicts of interest in this work.

\section{References}

1. Barbar SD, Binquet C, Monchi M, Bruyère R, Quenot JP. Impact on mortality of the timing of renal replacement therapy in patients with severe acute kidney injury in septic shock: the IDEAL-ICU study (initiation of dialysis early versus delayed in the intensive care unit): study protocol for a randomized controlled trial. Trials. 2014;15:270.

2. Mickells GE, Moge MA, Smith CM. Acute kidney injury in pediatric sepsis. Clin pediatr Emerg Med. 2014;15(2):185-192.

3. Bagshaw SM, George C, Bellomo R, Committee ADM. Early acute kidney injury and sepsis: a multicentre evaluation. Crit Care. 2008;12(2):R47.

4. Oppert M, Engel C, Brunkhorst FM, et al. Acute renal failure in patients with severe sepsis and septic shock - a significant independent risk factor for mortality: results from the German Prevalence Study. Nephrol Dial Transplant. 2008;23(3):904-909.

5. Prowle JR, Bellomo R. Sepsis-associated acute kidney injury: macrohemodynamic and microhemodynamic alterations in the renal circulation. Semin Nephrol. 2015;35(1):64-74.

6. Tökés-Füzesi M, Woth G, Ernyey B, et al. Microparticles and acute renal dysfunction in septic patients. J Crit Care. 2013;28(2):141-147.

7. Gocze I, Koch M, Renner P, et al. Urinary biomarkers TIMP-2 and IGFBP7 early predict acute kidney injury after major surgery. PLoS One. 2015;10(3): $\mathrm{e} 0120863$.

8. Majumdar A. Sepsis-induced acute kidney injury. Indian J Crit Care Med. 2010;14(1):14-21.

9. de Geus HR, Betjes MG, Bakker J. Biomarkers for the prediction of acute kidney injury: a narrative review on current status and future challenges. Clin Kidney J. 2012;5(2):102-108.

10. Mårtensson J, Martling CR, Oldner A, Bell M. Impact of sepsis on levels of plasma cystatin C in AKI and non-AKI patients. Nephrol Dial Transplant. 2012;27(2):576-581.

11. Dellinger RP, Levy MM, Rhodes A, et al. Surviving Sepsis Campaign Guidelines Committee including the Pediatric Subgroup. Surviving sepsis campaign: international guidelines for management of severe sepsis and septic shock: 2012. Crit Care Med. 2013;41(2):580-637.

12. Hoste EAJ, Blot SI, Lameire NH, Vanholder RC, De Bacquer D, Colardyn FA. Effect of nosocomial bloodstream infection on the outcome of critically Ill patients with acute renal failure treated with renal replacement therapy. J Am Soc Nephrol. 2004;15(2):454-462.

13. Ahmed W, Memon JI, Rehmani R, Juhaiman A. Outcome of patients with acute kidney injury in severe sepsis and septic shock treated with early goal-directed therapy in an intensive care unit. Saudi J Kidney Dis Transpl. 2014;25(3):544-551.

14. Alobaidi R, Basu RK, Goldstein SL, Bagshaw SM. Sepsis-associated acute kidney injury. Semin Nephrol. 2015;35(1):2-11.

15. Leedahl DD, Frazee EN, Schramm GE, et al. Derivation of urine output thresholds that identify a very high risk of AKI in patients with septic shock. Clin J Am Soc Nephrol. 2014;9(7):1168-1174.

16. Amin A, Nasr H, Younis G, Gamal H. Short term prognostic utility of Tc-99m DMSA renal imaging in sepsis induced acute renal failure; provisional data. Int J Clin Med. 2013;04(12):543-547.
17. McGuire TR, Reardon NT, Bogard K, et al. IL6 plasma concentrations in patients with sepsis receiving SLED and antibiotics: a predictor for survival. In Vivo. 2014;28(6):1131-1134.

18. Dabrowski W, Kocki T, Pilat J, Parada-Turska J, Malbrain ML. Changes in plasma kynurenic acid concentration in septic shock patients undergoing continuous veno-venous haemofiltration. Inflammation. 2014; 37(1):223-234.

19. Shapiro NI, Trzeciak S, Hollander JE, et al. The diagnostic accuracy of plasma neutrophil gelatinase-associated lipocalin in the prediction of acute kidney injury in emergency department patients with suspected sepsis. Ann Emerg Med. 2010;56(1):52 e1-59 e1.

20. Sood MM, Shafer LA, Ho J, et al. Early reversible acute kidney injury is associated with improved survival in septic shock. J Crit Care. 2014;29(5):711-717.

21. Brunkhorst FM, Engel C, Bloos F, et al. German Competence Network Sepsis (SepNet). Intensive insulin therapy and pentastarch resuscitation in severe sepsis. N Engl J Med. 2008;358(2):125-139.

22. Finfer S, McEvoy S, Bellommo R, McArthur C, Myburgh J, Norton R. SAFE Study Investigators . For the safe study investigators. Impact of albumin compared to saline on organ function and mortality of patients with severe sepsis. Intensive Care Med. 2011;37(1):86-96.

23. Perner A, Haase N, Guttormsen AB, et al. Hydroxyethyl starch 130/0.42 versus Ringer's acetate in severe sepsis. N Engl J Med. 2012;367(2): 124-134.

24. Myburgh JA, Finfer S, Bellomo R, et al. Hydroxyethyl starch or saline for fluid resuscitation in intensive care. $N$ Engl J Med. 2012;367(20): 1901-1911.

25. Young P, Bailey M, Beasley R, et al. Effect of a buffered crystalloid solution vs saline on acute kidney injury among patients in the intensive care unit: The SPLIT randomized clinical trial. JAMA. 2015;314(16): 1701-1710.

26. de Oliveira FS, Freitas FG, Ferreira EM, et al. Positive fluid balance as a prognostic factor for mortality and acute kidney injury in severe sepsis and septic shock. J Crit Care. 2015;30(1):97-101.

27. Kockara A, Kayatas M. Renal cell apoptosis and new treatment options in sepsis-induced acute kidney injury. Ren Fail. 2013;35(2):291-294.

28. Gordon AC, Mason AJ, Perkins GD, Ashby D, Brett SJ. Protocol for a randomised controlled trial of VAsopressin versus Noradrenaline as Initial therapy in Septic sHock (VANISH). BMJ Open. 2014;4(7):e005866.

29. Otto GP, Sossdorf M, Breuel H, et al. Renal outcome after vancomycin treatment and renal replacement therapy in patients with severe sepsis and septic shock: a retrospective study. J Crit Care. 2014;29(4):656-661.

30. Peng ZY, Wang HZ, Srisawat N, et al. Bactericidal antibiotics temporarily increase inflammation and worsen acute kidney injury in experimental sepsis. Crit Care Med. 2012;40(2):538-543.

31. Shum HP, Chan KC, Kwan MC, Yeung AW, Cheung EW, Yan WW. Timing for initiation of continuous renal replacement therapy in patients with septic shock and acute kidney injury. Ther Apher Dial. 2013;17(3):305-310.

32. Joannes-Boyau O, Honore PM, Perez P, et al. High-volume versus standard-volume haemofiltration for septic shock patients with acute kidney injury (IVOIRE study): a multicentre randomized controlled trial. Intensive Care Med. 2013;39(9):1535-1546.

33. Zhang P, Yang Y, Lv R, Zhang Y, Xie W, Chen J. Effect of the intensity of continuous renal replacement therapy in patients with sepsis and acute kidney injury: a single-center randomized clinical trial. Nephrol Dial Transplant. 2012;27(3):967-973.

34. Clark E, Joannes-Boyau O, Honoré PM, Sikora L, Bagshaw SM. Highvolume hemofiltration for septic acute kidney injury: a systematic review and meta-analysis. Crit Care. 2014;18(R7).

35. Borthwick EM, Borthwick CJH, Rabindranath KS, Maxwell AP, McAuley DF, Blackwood B. High-volume haemofiltration for sepsis. Cochrane Database of Syst Rev. 2013;1:CD008075.

36. Chancharoenthana W, Tiranathanagul K, Srisawat N, et al. Enhanced vascular endothelial growth factor and inflammatory cytokine removal with online hemodiafiltration over high-flux hemodialysis in sepsis-related acute kidney injury patients. Ther Apher Dial. 2013;17(5):557-563. 
37. Shum HP, Chan KC, Kwan MC, Yan WW. Application of endotoxin and cytokine adsorption haemofilter in septic acute kidney injury due to Gram-negative bacterial infection. Hong Kong Med J. 2013;19(6): 491-497.

38. Payen DM, Guilhot J, Launey Y, et al. Early use of polymyxin B hemoperfusion in patients with septic shock due to peritonitis: a multicenter randomized control trial. Intensive Care Med. 2015;41(6):975-984.

39. Tumlin J, Wali R, Williams W, et al. Efficacy and safety of renal tubule cell therapy for acute renal failure. J Am Soc Nephrol. 2008;19(5): 1034-1040.

40. Kaushal GP, Shah SV. Challenges and advances in the treatment of AKI. J Am Soc Nephrol. 2014;25(5):877-883.

41. Pickkers P, Heemskerk S, Schouten J, et al. Alkaline phosphatase for treatment of sepsis-induced acute kidney injury: a prospective randomized double-blind placebo-controlled trial. Crit Care. 2012; 16(1):R14.

42. Peters E, Arend J, Tiessen R, Van Elsas A, Masereeuw R, Pickkers P. Pharmacokinetics, safety and tolerability of human recombinant alkaline phosphatase in healthy volunteers. Critical Care. 2015; 19(Suppl 1):P126.

43. Heemskerk S, van Haren FM, Foudraine NA, et al. Short-term beneficial effects of methylene blue on kidney damage in septic shock patients. Intensive Care Med. 2008;34(2):350-354.

44. Swaminathan S, Rosner MH, Okusa MD. Emerging therapeutic targets of sepsis-associated acute kidney injury. Semin Nephrol. 2015; 35(1):38-54.

46. Schortgen F, Asfar P. Update in sepsis and acute kidney injury 2014. Am J Respir Crit Care Med. 2015;191(11):1226-1231.
46. Servillo G, Vargas M, Pastore A, et al. Immunomodulatory effect of continuous venovenous hemofiltration during sepsis: preliminary data. BioMed Res Int. 2013;108951:1-6.

47. Bi B, Schmitt R, Israilova M, Nishio H, Cantley LG. Stromal cells protect against acute tubular injury via an endocrine effect. J Am Soc Nephrol. 2007;18(9):2486-2496.

48. Heung M, Koyner JL. Entanglement of sepsis, chronic kidney disease, and other comorbidities in patients who develop acute kidney injury. Semin Nephrol. 2015;35(1):23-37.

49. Leelahavanichkul A, Huang Y, Hu X, et al. Chronic kidney disease worsens sepsis and sepsis-induced acute kidney injury by releasing High Mobility Group Box Protein-1. Kidney Int. 2011;80(11):1198-1211.

50. Huang X, Chen Y, Chung CS, et al. Identification of B7-H1 as a novel mediator of the innate immune/proinflammatory response as well as a possible myeloid cell prognostic biomarker in sepsis. J Immunol. 2014;192(3):1091-1099.

51. Yeh CH, Chou W, Chu CC, et al. Anticancer agent 2-methoxyestradiol improves survival in septic mice by reducing the production of cytokines and nitric oxide. Shock. 2011;36(5):510-516.

52. Jo SK, Rosner MH, Okusa MD. Pharmacologic treatment of acute kidney injury: why drugs haven't worked and what is on the horizon. Clin J Am Soc Nephrol. 2007;2(2):356-365.

53. Németh K, Leelahavanichkul A, Yuen PS, et al. Bone marrow stromal cells attenuate sepsis via prostaglandin E(2)-dependent reprogramming of host macrophages to increase their interleukin-10 production. Nat Med. 2009;15(1):42-49.

54. Zarjou A, Agarwal A. Sepsis and acute kidney injury. J Am Soc Nephrol. 2011;22(6):999-1006.
Biologics: Targets and Therapy

\section{Publish your work in this journal}

Biologics: Targets and Therapy is an international, peer-reviewed journal focusing on the patho-physiological rationale for and clinical application of Biologic agents in the management of autoimmune diseases, cancers or other pathologies where a molecular target can be identified. This journal is indexed on PubMed Central, EMBase, and Scopus.

\section{Dovepress}

The manuscript management system is completely online and includes a very quick and fair peer-review system, which is all easy to use Visit http://www.dovepress.com/testimonials.php to read real quotes from published authors. 